\title{
Adherence of bacteria to intraocular lenses: a prospective study
}

\author{
Aoife Doyle, Bijan Beigi, Anne Early, Alison Blake, Peter Eustace, Rosemary Hone
}

\begin{abstract}
Aims-The study was designed to investigate the bacterial flora of the operating field during routine cataract surgery and the source of intraocular lens contamination during the surgery.

Methods-The normal flora of the external eye and fornices of 17 patients undergoing selective cataract surgery was determined preoperatively. Swabs taken from the eyelid surface and lashes showed coagulase negative staphylococci (CNS) in 90\%, Propionibacterium acnes in $62 \%$, Corynebacterium sp in $18 \%$, and Peptostreptococcus in $3 \%$ of the patients. The lower fornices of $70 \%$ had CNS, 47\% P acnes, 6\% Staphylococcus aureus, $6 \%$ Corynebacterium sp, and $6 \%$ Candida.
\end{abstract}

Results-A sterile PMMA intraocular lens was touched on the upper bulbar conjunctiva immediately before the surgery. Eighty two per cent of lenses grew CNS, $18 \%$ P acnes, $18 \%$ Bacillus sp, $12 \% S$ aureus, and 6\% Corynebacterium sp. A second sterile PMMA intraocular lens was left on the drape and near the eye during surgery. Forty seven per cent of these cultured CNS, $12 \%$ Corynebacterium sp, and $6 \%$ Bacillus sp. A high count of bacteria in the operating field, especially CNS and $P$ acnes can contribute to postoperative inflammation and endophthalmitis.

Conclusion-Special measures are needed before and during the surgery to reduce the chance of intraocular inoculation of these bacteria. Use of proper culture media and techniques are necessary to identify these organisms, especially anaerobes, in postoperative inflammation.

(Br f Ophthalmol 1995; 79: 347-349)

University Colle

Misericordiae

Hospital, Dublin

Department of

Ophthalmology

A Doyle

B Beigi

A Early

A Blake

P Eustace

Department of Microbiology $\mathrm{R}$ Hone

Correspondence to: Professor Peter Eustace, Department of

Ophthalmology, University

College Dublin, Mater

Misericordiae Hospital,
Milege

Mublin 7, Ireland.

Accepted for publication 16 November 1994 e aim of this study we gate the bacterial flora of the operating field during routine cataract surgery and to study the source of IOL contamination during the surgery.

\section{Material and methods}

Seventeen patients, nine male and eight female and without an active infectious disease, were entered into the study. Their ages ranged from 22 to 82 (mean 75) years. Preoperatively, three cotton wool swabs moistened in sterile saline were used to sample the skin of the upper lid, the base of the lashes, and the lower fornix of each patient. This was performed in the ward.

Ocular preparation in theatre included cleaning of eyelids and lashes with chlorhexidine acetate BP $0.02 \%, 1 / 5000$ dilution. Lashes were not cut and no prophylactic topical or systemic antibiotics were used. Patients were draped with disposable $3 \mathrm{M}$ perforated ophthalmic sheet 1064 , and an adhesive barrier (3M steridrape 2035) was used to keep the lashes away from the field of the surgery.

Thirty four sterile PMMA IOLs were used in the operating theatre (none of the IOLs were irrigated before use). After draping each patient, the upper lid was pulled up and a sterile IOL was touched onto the upper bulbar conjunctiva using a sterile forceps (total 17). It was then placed in a sterile container and immediately transported to the laboratory.

Peroperatively, a control lens was placed on the drape within $2-4 \mathrm{~cm}$ of the edge of the steridrape (total 17). It was left in this position for the duration of surgery, 20 to 40 (mean 30 minutes). It was then placed in a sterile container and immediately sent to the laboratory.

Two settle plates, one containing Columbia agar with $7 \%$ horse blood and the other containing Lab $\mathrm{M}$ fastidious anaerobic agar with $7 \%$ horse blood and nalidixic acid, were exposed in the operating theatre in each instance to monitor airborne contamination.

Swabs and IOLs were cultured without delay, onto Columbia blood agar plates containing $7 \%$ horse blood, and onto Lab $\mathrm{M}$ fastidious anaerobic agar plates (FAA) containing $7 \%$ horse blood and nalidixic acid as a selective agent. Lenses were then dropped into cooked meat medium which was incubated at $37^{\circ} \mathrm{C}$. Blood agar plates were incubated at $37^{\circ} \mathrm{C}$ in $10 \%$ carbon dioxide; FAA plates were incubated at $37^{\circ} \mathrm{C}$.

All plates were examined after incubation for 48 hours, 1 week, and 2 weeks. Cooked meats were subcultured at 48 hours, 1 week, and 2 weeks. Each morphologically distinct colony was Gram stained.

Propionibacterium acnes was isolated on FAA medium after incubation for between 48 hours and 10 days. It was identified by the following characteristics: failure to grow aerobically, Gram positive rods on Gram stain, catalase positive, and biochemically by the rapID ANA 
Table 1 Micro-organisms identified at each site

\begin{tabular}{lcclll}
\hline & & & & $\begin{array}{l}\text { IOL touched } \\
\text { on to the } \\
\text { conjunctiva }\end{array}$ & $\begin{array}{l}\text { IOL left on } \\
\text { the drape }\end{array}$ \\
Organism & Eyelids & Eyelashes & Fornices & & \\
\hline Coagulase negative staphylococci & $17(100 \%)$ & $12(70 \%)$ & $12(70 \%)$ & $14(82 \%)$ & $8(47 \%)$ \\
Staphylococcus aureus & 0 & 0 & $1(6 \%)$ & $2(12 \%)$ & 0 \\
Corynebacterium sp & $1(6 \%)$ & $5(30 \%)$ & $1(6 \%)$ & $1(6 \%)$ & $2(12 \%)$ \\
Bacillus sp & 0 & 0 & 0 & $3(18 \%)$ & $1(6 \%)$ \\
Propionibacterium acnes & $12(70 \%)$ & $8(47 \%)$ & $8(47 \%)$ & $3(18 \%)$ & 0 \\
Peptostreptococcus & $1(6 \%)$ & 0 & 0 & 0 & 0 \\
Candida & 0 & 0 & $1(6 \%)$ & 0 & 0 \\
\hline
\end{tabular}

system (Innovative Diagnostic System Inc). Gram positive cocci isolated on blood agar plates were identified by the coagulase test as Staphylococcus aureus or coagulase negative staphylococci (CNS). Anaerobic Gram positive cocci isolated only on FAA were identified as Peptostreptococcus by the ID8 Mastring.

Gram positive bacilli which grew both aerobically and anaerobically were identified morphologically as Corynebacterium sp. Gram positive, oxidase positive bacilli were identified as Bacillus sp. Any new growth of staphylococci or corynebacteria from the cooked meat subcultured at 2 weeks was disregarded as a contaminant if not present in the initial subcultures or on the original plates.

One isolate of $P$ acnes from each patient was tested for sensitivity to 19 antibiotics (Table 3). Oxford $S$ aureus NCTC 6571 was used as a control.

\section{Results}

Studying the swabs taken from the skin of the eyelids, all 17 patients $(100 \%)$ had CNS, $12(70 \%)$ had $P$ acnes, one $(6 \%)$ had Corynebacterium, one $(6 \%)$ had Peptostreptococcus. Culture of the swabs from eyelashes showed 12 patients with CNS (70\%), eight with $P$ acnes (47\%), five with Corynebacterium (30\%). Swabs of the conjunctival sac grew CNS in 12 patients $(70 \%)$, $P$ acnes in eight (47\%), $S$ aureus in one $(6 \%)$, Corynebacterium in one (6\%), and Candida in one $(6 \%)$ patient (Table 1$)$.

Table 2 Rate of positive cultures from each source

\begin{tabular}{lll}
\hline Source & Numbers & Percentage (\%) \\
\hline Upper eyelid skin & $16 / 17$ & $94 \cdot 1$ \\
Eyelashes & $14 / 17$ & $82 \cdot 4$ \\
Conjunctival sac & $13 / 17$ & $76 \cdot 5$ \\
Lens touched on to the bulbar & & \\
$\quad$ conjunctiva & $15 / 17$ & $88 \cdot 2$ \\
Lens left on the drape & $11 / 17$ & $64 \cdot 7$ \\
\hline
\end{tabular}

Table 3 Antibiotic sensitivity of Propionibacterium acnes

\begin{tabular}{ll}
\hline Sensitive to & Resistant to \\
\hline Ampicillin & Amikacin \\
Cefaclor & Ciprofloxacin \\
Cefotaxime & Gentamicin \\
Cephalothin & Metronidazole \\
Cephradine & Netilmicin \\
Chloramphenicol & Oxacillin (7/15) \\
Erythromycin & Trimethoprim \\
Fucidic acid & \\
Oxacillin (8/15) & \\
Penicillin & \\
Teicoplanin & \\
Tetracycline & \\
Vancomycin & \\
\hline
\end{tabular}

From 15 positive cultures, eight were sensitive and seven were resistant to oxacillin.
Results of cultures taken from the 17 lenses touched onto the upper bulbar conjunctiva were as follows: CNS in 14 patients (82\%), Bacillus species in three (18\%), $P$ acnes in three $(18 \%), S$ aureus in two $(12 \%)$, and Corynebacterium in one (6\%) patient (Table 1).

Interestingly from 17 lenses left on the drape during the surgery, 18 (47\%) grew CNS, two $(12 \%)$ Corynebacterium, and one (6\%) Bacillus species (Table 1).

The rate of the positive cultures from each source are shown in Table 2 . At least $70 \%$ of cultures were positive. Settle plates during each operation yielded low colony count which included small numbers of CNS (less than two colony forming units per square foot per minute). $P$ acnes was not found as an airborne contaminant. Table 3 shows the sensitivity of $P$ acnes isolated for each patient. Staphylococci growth was not tested against a wide spectrum of antibiotics but they were universally sensitive to vancomycin.

\section{Discussion}

CNS can be cultured in less than 24 hours in the laboratory. It has a high presence in the normal flora of the skin. It has been shown to adhere to the IOL by direct contact or exposure to air. ${ }^{4}$

In this study CNS was present in the external eyes of all patients. In $70 \%$ CNS was isolated both from the external eye and from the fornices. Eighty per cent of the lenses in contact with the bulbar conjunctiva and almost half of the control lenses left on the drape during the surgery were also contaminated by this organism. This can cause an early and in rare occasions a late onset postoperative infection after insertion of the IOL. ${ }^{3} S$ aureus was present in one patient's fornix and grew from two IOLs in contact with the conjunctiva. Its role in postoperative endophthalmitis is not disputed.

The high prevalence of $P$ acnes in the operating field of patients in this study is of particular interest considering the recently known role of Propionibacterium in postoperative uveitis. ${ }^{6}$ $P$ acnes was isolated from one or more sites in all but two patients in this study, a much higher prevalence than in previous studies. ${ }^{78}$ This may be due to the fact that each sample was immediately dealt with in accordance with the recommendations made by Meisler $e t a l$ for the isolation of anaerobes. ${ }^{9} P$ acnes tends to loculate within the capsular bag after cataract surgery. It may cause a persistent and relatively indolent inflammation. ${ }^{10}$ Of 17 patients in whom the IOL was touched onto the conjunctiva, three IOLs were tested positive for $P$ acnes $(18 \%)$. Postoperative uveitis developed in one of them.

Although the incidence of intraocular infection by Propionibacterium has been estimated to be as low as $0.086-0.51 \%$, this would be significant when one considers both the number of patients undergoing cataract surgery and the severity of the complications. Propionibacterium endophthalmitis can present from 2-10 months after the surgery. ${ }^{9}$ Hence, in case of 
late onset endophthalmitis, special effort should be made to isolate propionibacteria. Even under optimal anaerobic conditions in the laboratory it takes 2 to 7 days to culture Propionibacterium. The sensitivity and resistance of $P$ acnes to antibiotics shown in Table 3, corresponds with previous studies. ${ }^{8}$

There is no doubt that the eyelashes and the conjunctival sac can be a source of contamination of the IOL by direct contact. Andreason et $a l^{10}$ have suggested the use of a sterile adhesive barrier between the surgical field and the eyelids and facial skin. They have also proposed irrigation of the conjunctival sac with a sterile $0.9 \%$ saline solution just before surgery. However, it is possible that the irrigation of the conjunctival sac may wash organisms hidden within the surface crypts of the conjunctiva to the operation field where they remain. ${ }^{11}$

It is significant that the lenses left on the sterile drape for the duration of surgery picked up bacteria from the air in the theatre in 11 out of 17 cases. Bacterial cell count in theatre on the same day was within an acceptable level.

The electrostatic surface charge of the IOL attracts the bacteria. Therefore, it might be appropriate to incorporate a broad spectrum antibiotic into irrigation fluid used during cataract surgery. ${ }^{12}$ We have used gentamicin routinely for such purposes $(0.8 \mathrm{mg} / 100 \mathrm{ml})$. We have now added vancomycin $(2 \mathrm{mg} / 100 \mathrm{ml})$ for a more appropriate cover (Table 3).

In this study the presence of $S$ aureus, CNS, and $P$ acnes in the operating field of the patients undergoing standard cataract surgery has been shown. These can be the source of intraocular infection either by contamination of the $\mathrm{IOL}^{13}$ or direct intraocular inoculation. Staphylococci can cause an early, and Propionibacterium a late onset endophthalmitis. Microbes of the external eye and fornices by direct contact and organisms in the air attracted by the electrostatic surface charge of the IOL can cause postoperative inflammation and infection. Careful preoperative preparation of the external eye, use of sterile adhesive barriers to keep the eyelashes away from the field of the surgery, and use of appropriate antibiotics in the infusion solution have been suggested. Special attempts should be made to identify the above organisms, especially anaerobes, in postoperative inflammation.

We are grateful to Mrs Christina Yeardsley and Ms Norita O'Connor for their help.

1 Apple DJ, Mammalis N, Steinmets RL, Loftfield K, Crandall AS, Olson J. Phacoanaphylactic endophthalmitis associated with extracapsular cataract extraction and posterior chamber intraocular lens. Arch Ophthalmol 1984; 102: 1528-32.

2 Meltzer DW. Sterile hypopyon following intraocular lens surgery. Arch Ophthalmol 1980; 98: 100-4.

3 Stern GA, Engel HM, Driebe Jr W. Recurrent post-operative endophthalmitis. Cornea 1990; 9: 102-7.

4 Vafidis GA, Marsh RJ, Stacey AR. Bacterial contamination of intraocular lens surgery. $\mathrm{Br} \mathcal{F}$ Ophthalmol 1984; 68: 520-3.

5 Jansen B, Hartman C, Perdreau FS, Peters G. Late onset endophthalmitis associated with intraocular lens: a case of molecularly proved Staph epidermidis aetiology. $\mathrm{Br} \mathcal{f}$ Ophthalmol 1991; 75: 440-1.

6 Roussel TJ, Culbertson WW, Jaffe NS. Chronic postoperative endophthalmitis associated with Propionibacterium tive endophthalmitis associated with Propion
acnes. Arch Ophthalmol 1987; 105: 1199-201.

7 McNatt J, Allan SD, Wilson LA, Dowell VE Jr. Anaerobic flora of the normal human conjunctival sac. Arch Ophthalmol 1978; 96: 1448-50.

8 Wang WLL, Everett ED, Johnson M, Dean E. Susceptibility of Propionibacterium acnes to 17 antibiotics. Antimicrob Agents Chemotherapy 1977; 11: 171-3.

9 Meisler DM, Mandelbaum S. Propionibacterium-associated endophthalmitis after extra-capsular cataract extraction. Ophthalmology 1989; 96: 54-9.

10 Andreason H, Alsbirk KE, Schonheyder HC, Anderson CU. Propionibacterium acnes in the eyes of cataract CU. Propionibacterium acnes in the eyes of
patients. Eur $\mathcal{F}$ Implant Ref Surg 1992; 4: 83-5.

11 Isenburg C, Apt L, Yoshimuri R. Chemical preparation of the eye in ophthalmic surgery. Arch Ophthalmol 1983; 101: 761-3.

12 Gill JP. Filtres and antibiotics in irrigating solution for cataract surgery. 7 Cataract Refract Surg 1991; 17: 385.

13 Spencer SR, Dealler SF, Hassett PDA, Todd NJ, Hawkey PM, Noble BA. Bacterial contamination of intraocular lenses: the source of the bacteria. Eye $1989 ; 3: 685-9$. 